\title{
Universal fluctuations in heavy-ion collisions in the Fermi energy domain
}

\author{
R. Botet ${ }^{\dagger}$, M. Płoszajczak ${ }^{\ddagger}$, A. Chbihi ${ }^{\ddagger}$, B. Borderie $^{\S}$, D. Durand ${ }^{\&}$, J. Frankland ${ }^{\ddagger}$ \\ $\dagger$ Laboratoire de Physique des Solides - CNRS, Bâtiment 510, Université Paris-Sud, Centre d'Orsay, F-91405 Orsay, France \\ ¥ Grand Accélérateur National d'Ions Lourds (GANIL), CEA/DSM-CNRS/IN2P3, BP 55027, F-14076 Caen Cedex, France \\ $\S$ Institut de Physique Nucléaire, IN2P3-CNRS, F-91406 Orsay Cedex, France \\ \& LPC, IN2P3-CNRS, ISMRA et Université, F-14050 Caen Cedex, France
}

(November 13, 2018)

\begin{abstract}
We discuss the scaling laws of both the charged fragments multiplicity $n$ fluctuations and the charge of the largest fragment $Z_{\max }$ fluctuations for $X e+S n$ collisions in the range of bombarding energies between $25 \mathrm{~A} \cdot \mathrm{MeV}$ and $50 \mathrm{~A} \cdot \mathrm{MeV}$. We show at $E_{l a b} \gtrsim 32 \mathrm{MeV} / \mathrm{A}$ the transition in the fluctuation regime of $Z_{\max }$ which is compatible with the transition from the ordered to disordered phase of excited nuclear matter. The size (charge) of the largest fragment is closely related to the order parameter characterizing this process.
\end{abstract}

Theoretical description of the fragment production in heavy-ion (HI) collisions depends on whether the equilibrium has been reached before the system starts fragmenting. Possibility of the critical behavior associated with the transition from the particle evaporation regime at low excitation energies to the explosion of the hot source at about $5-10 \mathrm{MeV} /$ nucleon cannot be excluded. Unfortunately, this exciting possibility is difficult to study because all standard models and methods of characterizing different phases and transitions of the nuclear matter in HI collisions assume an equilibrium mechanism of the fragment production. In this work, we shall apply new methods of the theory of universal fluctuations of observables in finite systems 11 to examine what can be said in a model independent way about the fragmentation mechanism and the phase changement in HI collisions in the Fermi energy domain. Our analysis, which is independent of the assumption of the equilibrium in the fragments production process, uses the data of the INDRA multidetector system for $X e+S n$ collisions at $25 \mathrm{MeV} \leq E_{l a b} / A \leq 50 \mathrm{MeV}$ [2]5].

Several features of finite systems are important if one wants to study either the criticality or the distance to the critical point [1]. These are :

(i) The $\Delta$-scaling of the normalized probability distribution $P_{<m>}[m]$ of the variable $m$ for different 'system sizes' $<m>$ :

$$
\begin{aligned}
<m>^{\Delta} P_{<m>}[m] & =\Phi\left(z_{(\Delta)}\right), \quad 0<\Delta \leq 1 \\
z_{(\Delta)} & =\left(m-m^{*}\right) /<m>^{\Delta},
\end{aligned}
$$

where $\left\langle m>\right.$ and $m^{*}$ are the average and the most probable values of $m$ respectively, and $\Phi\left(z_{(\Delta)}\right)$ is the positive defined scaling function which depends only on a single scaled variable $z_{(\Delta)}$. If the scaling framework holds, the scaling relation (1) is valid independently of any phenomenological reasons for changing $\langle m>[1]$. The scaling domain in (1) is defined by the asymptotic behaviour of $P_{<m>}[m]$ when $m \rightarrow \infty$ and $<m>\rightarrow \infty$, but $z_{(\Delta)}$ has a finite value. The $\Delta$-scaling analysis is very robust and can be studied even in small systems if the probability distributions $P_{<m>}[m]$ are known with a sufficient precision. In small systems however, the value of scaling parameter $\Delta$ may differ slightly from its asymptotic value [1] ;

(ii) The tail of the scaling function $\Phi\left(z_{(\Delta)}\right)$ and the anomalous dimension $\mathrm{g}$.

All these features are related to the properties of the scaling function which characterizes the finite system.

If the infinite system experiences a second-order phase transition, and if $m$ is the extensive (scalar) order parameter then [1] :

(i) At the critical point, the corresponding finite system exhibits the 'first-scaling law' $(\Delta=1)$ and the tail of scaling function for large positive $z_{(\Delta)}: \Phi\left(z_{(\Delta)}\right) \sim$ $\exp \left(-z_{(\Delta)}^{\tilde{\nu}}\right)$, is characterized by a large value of the exponent $\tilde{\nu}=1 /(1-g)>2$.

(ii) The finite system exhibits the 'second-scaling law' $(\Delta=1 / 2)$ in the ordered phase, and the first-scaling law in the disordered phase. In both cases, the tail of the scaling function is gaussian $(\tilde{\nu}=2)$. Close to the critical point, one may find also the cross-over phenomenon from the first-scaling to the second-scaling law by the continuous $\Delta$-scaling law, again with the gaussian tail of the scaling function.

If the parameter $m$ is not singular at the transition, then its probability distribution follows the secondscaling law with the gaussian tail.

There are two generic families of the fragment production scenarios for which the second-order phase transition has been identified. The family of aggregation scenarios contains both equilibrium models like the Fisher droplet model, the Ising model or the percolation model, and off-equilibrium models like the Smoluchowski model of gelation. In these models, the average size of the largest cluster $\left\langle s_{\max }\right\rangle$ is the order parameter [1, 6] and the exponent $\tau$ of the power-law cluster-size distribution at the critical point $(\tau>2): n(s) \sim s^{-\tau}$, is related to the 
anomalous dimension as : $g=1 /(\tau-1)$. The second family includes fragmentation scenarios and contains
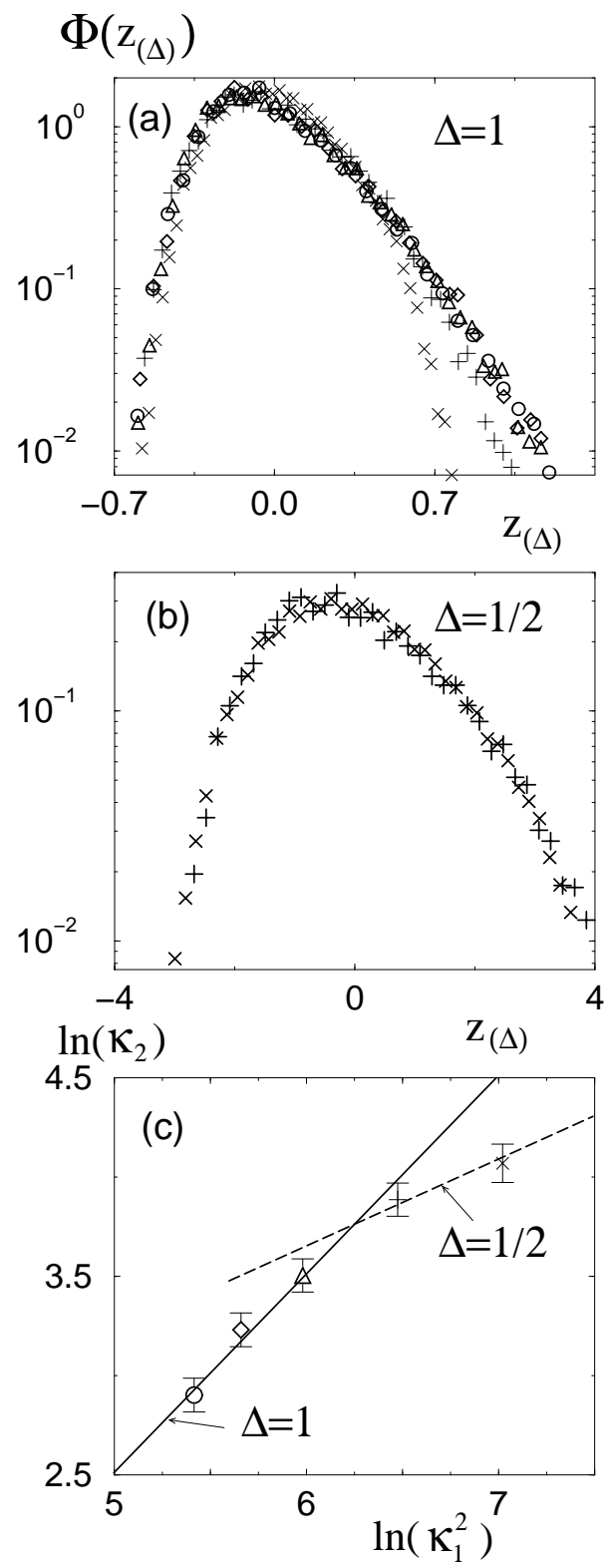

FIG. 1. Different characteristics of the largest fragment charge probability distributions $P\left[Z_{\max }\right]$ for central $\mathrm{Xe}+\mathrm{Sn}$ collisions. (a) $P\left[Z_{\max }\right]$ in the variables of the first-scaling law for $E_{l a b} / A=25$ (asterisks), 32 (crosses), 39 (triangles), 45 (diamonds) and 50 (circles) $M e V$. (b) $P\left[Z_{\max }\right]$ in the variables of the second-scaling law for $E_{l a b} / A=25$ and $32 \mathrm{MeV}$. (c) The normalized second cumulant moment $\mathcal{K}_{2}\left(\equiv \kappa_{2} / \kappa_{1}{ }^{2}\right)$ of $P\left[Z_{\max }\right]$ is plotted as a function of $\ln \left\langle Z_{\max }>^{2}\left(\equiv \ln \kappa_{1}^{2}\right)\right.$ together with the statistical error bars. The lines $\Delta=1$ and $\Delta=1 / 2$ are shown to guide the eyes.

various hybrids of the fragmentation - inactivation - binary model [7]:8]. In this family of models, the average fragment multiplicity $\langle n\rangle$ is the order parameter of the critical shattering process and the exponent $\tau$ at the critical point $(\tau<2)$ is related to the anomalous dimension as : $g=\tau-1$. The order parameters for these two different scenarios are not only relevant in the study of phase changes in $\mathrm{HI}$ collisions but they are also measurable. In this work, we shall investigate the patterns of the largest fragment charge distribution $P\left[Z_{\max }\right]$ and the charged fragment multiplicity distribution $P[n]$ for different centrality conditions and bombarding energies in the Fermi energy domain, using the methods of the theory of universal fluctuations of observables in finite systems [1].

Fig. 1 shows the $\Delta$-scaling features of $P\left[Z_{\max }\right]-$ distributions for central $\mathrm{Xe}+\mathrm{Sn}$ collisions at $25 \mathrm{MeV} \leq$ $E_{l a b} / A \leq 50 \mathrm{MeV}$. In the experiment a great effort was done to well identify in charge the different fragments produced and especially the heaviest ones [9, 10]. For each collision energy about 20000 events are taken into account in the present analysis. These events are selected with the experimental centrality condition : complete events (i.e., more than $80 \%$ of the total charge and momentum is detected) and $\Theta_{\text {flow }} \geq \pi / 3$. The latter quantity is a global observable defined as the angle between the beam direction and the main emission direction of matter in each event, which is determined from the energy tensor. It has been shown for the reactions in the Fermi energy domain that events with small $\Theta_{\text {flow }}$ are dominated by binary dissipative collisions [11, 12, 3]. For events with little or no memory of the entrance channel, $\Theta_{\text {flow }}$ is isotropically distributed. The upper part of the figure (Fig. 1a) shows that $P\left[Z_{\max }\right]$-distributions for $E_{l a b} / A=39,45,50 \mathrm{MeV}$ can be compressed into a single curve in the scaling variables of the first-scaling. The distributions for 25 and $32 \mathrm{MeV}$, which show strong deviations with respect to this scaling curve both near the maximum and in the tail for large $z_{(\Delta)}$, can be compressed into another single curve in the variables of the second-scaling, as shown in Fig. 1b. It should be stressed that we do not optimize the value of $\Delta$ because of the experimental (number of events) and theoretical (smallness of the system) limits, but we study whether the data is consistent with one of the two limits : $\Delta=1 / 2$ and 1 , which have a particular significance in the scaling theory of phase-transitions.

More straightforward global measure of scaling features is provided by the cumulant moments : $\kappa_{1}=<$ $m>, \kappa_{2}=<m^{2}>-<m>^{2}, \kappa_{3}=<m^{3}>-3<$ $m^{2}><m>+2<m>^{3}$, etc. In case of the $\Delta$-scaling, normalized cumulant moments : $\mathcal{K}_{q}^{(\Delta)} \equiv \kappa_{q} /\left(\kappa_{1}\right)^{q \Delta}$, are independent of the system size $\langle m>$ [1]. $\log$ of the normalized cumulant moment $\mathcal{K} \equiv \mathcal{K}_{2}^{(\Delta=1)}$ of $P\left[Z_{\text {max }}\right]$ is plotted in Fig. 1c versus $\log$ of $\left\langle Z_{\max }\right\rangle^{2}$, i.e. versus $\log$ of $\left(\kappa_{1}\right)^{2}$. The data for different $\left\langle Z_{\max }\right\rangle$, i.e. for different bombarding energies, should lie on a straight line if the $\Delta$-scaling holds. The slope of this line gives the value of $\Delta$. It is seen that the higher energy branch $\left(E_{l a b} / A=39,45,50 \mathrm{MeV}\right)$ follows the 
line $\Delta=1$ (the solid line), in agreement with Fig. 1a. The point for $E_{l a b} / A=25 \mathrm{MeV}$ is clearly off this line. The point for $E_{l a b} / A=32 \mathrm{MeV}$ aligns along the line $\Delta=1 / 2$ (the dashed line) passing through the point for $E_{l a b} / A=25 \mathrm{MeV}$, but is also close to the line $\Delta=1$. In this 'near-crossing' case, one has to investigate higher order moments, viz the whole probability distribution. Fortunately, Figs. 1a, 1b show clearly that the collisions at $32 \mathrm{MeV} / \mathrm{A}$ belong to the branch $\Delta=1 / 2$.

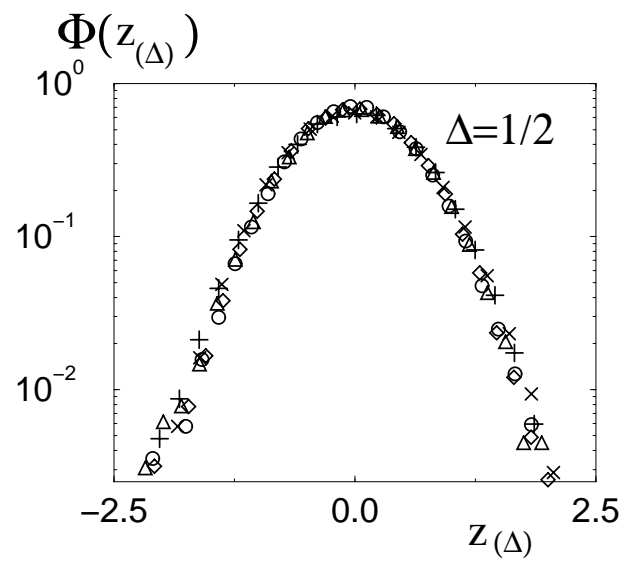

FIG. 2. The multiplicity distributions of charged fragments for central $\mathrm{Xe}+\mathrm{Sn}$ collisions are plotted in the variables of the second-scaling law. Notation of data at different collision energies is the same as in Fig. 1.

The pattern of charged fragment multiplicity distributions $P[n]$ does not show any significant evolution with the bombarding energy (Fig. 2), and the data is perfectly compressible in the scaling variables of the second-scaling, i.e. the multiplicity fluctuations are small $\left(\kappa_{q} \sim\left(\kappa_{1}\right)^{q / 2}\right)$ in the whole studied range of bombarding energies. The scaling features of experimental $P\left[Z_{\max }\right]-$ and $P[n]-$ probability distributions in Figs. 1 and 2 are complementary and allow to affirm that the fragment production in central HI reactions in the Fermi energy domain follows the aggregation scenario and exhibits the transition at $E_{l a b} / A \gtrsim 32 \mathrm{MeV}$ between the two phases of excited nuclear matter with distinctly different patterns of $Z_{\max }-$ fluctuations.

Assuming that the change of the fragmentation regime is controlled by the source excitation energy, one may ask to which extent similar scaling features and transitions can be seen in more peripheral collisions. For that purpose, we have investigated a sample of events selected with the experimental condition : complete events and $\Theta_{\text {flow }} \geq \pi / 18$. This sample contains mostly events of semi-central binary collisions which keep some memory of the entrance channel as manifested by the presence of two contributions in the velocity distribution of $Z_{\max }$ corresponding to the quasi-projectile and quasi-target [5]. Fig. 3a shows that $Z_{\max }$-distributions for $E_{l a b} / A=45$ and $50 \mathrm{MeV}$ can be compressed into a single curve in the variables of the first-scaling. The distributions for
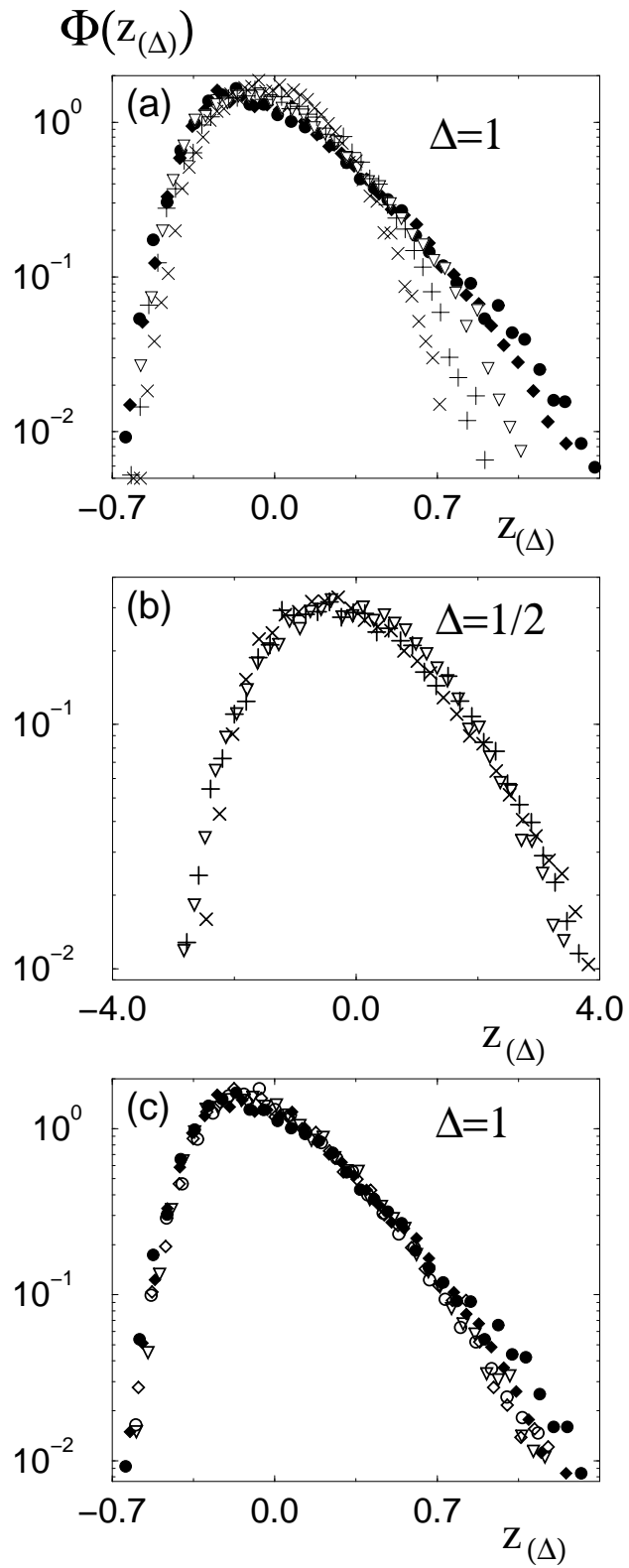

FIG. 3. Different characteristics of the largest fragment charge distributions $P\left[Z_{\max }\right]$ for $X e+S n$ collisions. (a) $P\left[Z_{\max }\right]$ for semi-central collisions in the variables of the first-scaling law for $E_{l a b} / A=25$ (asterisks), 32 (crosses), 39 (triangles), 45 (full diamonds) and 50 (full circles) $\mathrm{MeV}$. (b) $P\left[Z_{\text {max }}\right]$ for semi-central collisions at $E_{l a b} / A=25,32$ and 39 $\mathrm{MeV}$ in the variables of the second-scaling law. (c) $P\left[Z_{\max }\right]$ for semi-central collisions at $E_{l a b} / A=45$ and $50 \mathrm{MeV}$ and central collisions at $E_{l a b} / A=39,45$ and $50 \mathrm{MeV}$ in the variables of the first-scaling law. The notation for central collisions is the same as in Fig. 1.

25, 32 and $39 \mathrm{MeV}$ show significant deviations with respect to this scaling curve, both at the maximum and in the large- $z_{(\Delta)}$ tail. These three distributions are shown in Fig. $3 \mathrm{~b}$ in the variables of the second-scaling. 
$P\left[Z_{\text {max }}\right]$-distributions for $E_{l a b} / A=25$ and $32 \mathrm{MeV}$ can be perfectly compressed into a single curve. The data for $E_{\text {lab }} / A=39 \mathrm{MeV}$ is close to this curve but, nevertheless, shows some deviations for $z_{(1 / 2)} \simeq 1$. This data, which seems to be intermediate between the scaling limits $\Delta=1 / 2$ and $\Delta=1$, could indicate a continuous change of $\Delta$ in the transition region. There are presently not enough data to verify this possibility. We note however, that an optimal data compression for $E_{l a b} / A=32$ and $39 \mathrm{MeV}$ would indicate $\Delta \simeq 0.6$ for the latter one.

The scaling pattern of $P\left[Z_{\max }\right]$ in semi-peripheral collisions follows a similar general evolution with the bombarding energy (the excitation energy) as seen in central collisions, except that the branch $\Delta=1$ in semi-central collisions starts at higher energies than in central collisions. Fig. 3c presents all $Z_{\max }$-distributions which in semi-central and in central collisions belong to the respective first-scaling branches. It is non-trivial that these probability distributions, which correspond to different selection criteria, collapse approximately into the unique scaling curve. This example should encourage further investigation of the feasibility of semi-central events in this kind of studies. One should also note that the scaling functions in the branch $\Delta=1 / 2$ are essentially identical in semi-central and central collisions (compare Figs. 1b and $3 b)$.

The characteristic feature of critical behavior is the anomalous tail of the scaling function with $\tilde{\nu}>2$. Using all $P\left[Z_{\max }\right]$ shown in Fig. 3c, we have fitted the scaling function $\Phi\left(z_{(1)}\right)$ for $z_{(1)}>0$ by : $a \exp \left(-b\left(z_{(1)}-z_{0}\right)^{\tilde{\nu}}\right)$, where $z_{0}$ is the estimate of the most probable value of the distribution and $a, b, \tilde{\nu}$ are the fitting parameters. We have found : $\tilde{\nu}=1.6 \pm 0.4$, what is incompatible with typical values $(3.5 \lesssim \tilde{\nu} \lesssim 6)$ in the critical region for aggregation scenarios [1]. The same procedure for central and semi-central collisions in the second-scaling phase yields : $\tilde{\nu}=1.8 \pm 0.4$. Hence, the existing data do not show the critical bahavior in the transition region from ordered to disordered phase.

In conclusion, we have applied the theory of universal fluctuations in the finite systems [1] to the symmetric HI reactions in the Fermi energy domain. This theory provides rigorous methods to characterize critical and off-critical behaviors both in equilibrium and offequilibrium finite systems. This is an important novel aspect of the present analysis. Convincing scaling behavior of the $Z_{\max }-$ and $n$-distributions have been found in central collisions. The present analysis shows that the fragment production in central HI collisions at around the Fermi energy is governed by the aggregation scenario with $\left\langle Z_{\max }\right\rangle$ as the order parameter. The changement of the regime of $Z_{\max }$-fluctuations from the secondscaling at low energies to the first-scaling at higher energies, with the gaussian tail of the scaling function in both regimes, is compatible with the transition from the ordered phase to the disordered phase. The scaling curves determined from central and semi-central collisions are the same but the bombarding energy corresponding to change in the scaling behavior is higher for more peripheral collisions, in according with the expected stronger contribution of non-equilibrium effects in these collisions. The critical region, if exists in the nuclear fragmentation process, should be searched for in the narrow window of bombarding energies close to : $E_{l a b} / A \sim 32 \mathrm{MeV}$. Its signature would be the anomalous tail $\tilde{\nu}>2$ of $P\left[Z_{\text {max }}\right]$, in case of the second-order phase transition, or the double-hump shape of $P\left[Z_{\max }\right]$-distribution for the first-order phase transition [1]. Future studies of symmetric $\mathrm{HI}$ reactions, performed in small steps of bombarding energies, will hopefully allow to distinguish between the cross-over phenomenon and the other two scenarios which invoke existence of the phase-transition.

\section{Acknowledgements}

We are grateful to the Collaboration INDRA for providing us the experimental data. We thank J.-L. Charvet and J. Colin for stimulating discussions.

[1] R. Botet and M. Płoszajczak, Phys. Rev. E 62, 1825 (2000); Nucl. Phys. B (Suppl.) 92 (2001) no. 1-3; R. Botet and M. Płoszajczak, in Proc. of the 8th Int. Workshop on Multiparticle Production CORRELATIONS AND FLUCTUATIONS '98, T. Csörgo, S. Hegyi, G. Jancsó and R.C. Hwa eds., Matrahaza, Hungary, 14-21 June 1998, World Scientific (1999) 421; R. Botet and M. Płoszajczak, in Proc. XXVIII Int. Symposium on MULTIPARTICLE DYNAMICS, N.G. Antoniou, A.P. Contogouris, F.K. Diakonos, C.N. Ktorides, M. Stassinaki and M. Vassiliou eds., Delphi, Greece, 611 September 1998, World Scientific (2000) 217.

[2] R. Bougault et al. (INDRA Collaboration), in Proc. XXXV Int. Winter Meeting on Nuclear Physics, ed. by I. Iori , Bormio, (1997) 251.

[3] N. Marie et al. (INDRA Collaboration), Phys. Lett. B 391, 15 (1997).

[4] A. Le Fèvre, PhD-Thesis, GANIL T 9703.

[5] S. Salou, PhD-Thesis, GANIL T 9706.

[6] R. Botet and M. Płoszajczak, Journal of Sol-Gel Science and Technology 15, 167 (1999).

[7] R.M. Ziff and E.D. McGrady, J. Phys. A 18, 3027 (1985); E.D. McGrady and R.M. Ziff, Phys. Rev. Lett. 58, 892 (1987).

[8] R. Botet and M. Płoszajczak, Phys. Rev. Lett. 69, 3696 (1992); Phys. Rev. E 57, 7305 (1998).

[9] G. Tabacaru et al. (INDRA Collaboration), Nucl. Inst. and Meth. A 428, 379 (1999).

[10] M. Parlog et al. (INDRA Collaboration), Nucl. Inst. and Meth. A, to appear.

[11] J. Cugnon and D. L'Hote, Nucl. Phys. A 397, 512 (1983).

[12] J.F. Lecolley et al., Phys. Lett. B325, 317 (1994). 